\title{
Successful Endovascular Revascularization of Acute Basilar Artery Occlusion Approached via an Aberrant Right Subclavian Artery
} Case Report

Yoshiki Obata, Akihito Sato, and Masaru Aoyagi

Objective: We report a rare case of endovascular treatment for acute basilar artery occlusion (BAO) approached via the aberrant right subclavian artery (ARSA).

Case Presentation: A 66-year-old woman with atrial fibrillation was admitted for nausea and vomiting. She was progressively losing consciousness. At admission, her National Institutes of Health Stroke Scale (NIHSS) score was 38. Magnetic resonance angiography revealed BAO. An intravenous thrombolysis agent was administered at 2 hours and 20 minutes after symptom onset. Thrombectomy was performed using a stent retriever, resulting in complete recanalization at 4 hours and 39 minutes after symptom onset. While approaching the lesion, an ARSA was found. After the recanalization, her consciousness significantly improved, and her NIHSS score improved from 38 to 6 the day after the operation. She was discharged on day 17 with an NIHSS score of 1.

Conclusion: BAO is considered to have a poor prognosis. However, stent retriever thrombectomy may improve the prognosis of acute BAO. In addition, many anomalies such as ARSA can hinder the endovascular approach to intracranial arteries. Therefore, in-depth knowledge of vascular anatomy is necessary to perform this procedure.

Keywords > acute basilar artery occlusion, aberrant right subclavian artery, endovascular treatment

\section{Introduction}

Basilar artery occlusion (BAO) is believed to have a poor prognosis, with a mortality rate as high as $90 \%{ }^{1-3)}$ Because of the lack of prospective studies on acute BAO, the natural history, prognosis, and treatment of BAO have not been fully understood. ${ }^{2,4)}$

Generally, the treatment of acute BAO involves intravenous administration of recombinant tissue plasminogen activator (rt-PA). However, because intravenous rt-PA has been ineffective in most cases of acute BAO, endovascular

Department of Neurosurgery, Shioda Memorial Hospital, Choseigun, Chiba, Japan

Received: October 11, 2016; Accepted: March 27, 2017

Corresponding author: Yoshiki Obata. Department of Neurosurgery, Shioda Memorial Hospital, 550-1 Kouri, Nagara-machi, Chosei-gun, Chiba 297-0203, Japan

Email: obata-tmd@umin.ac.jp

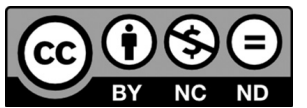

This work is licensed under a Creative Commons Attribution-NonCommercialNoDerivatives International License.

(C)2017 The Japanese Society for Neuroendovascular Therapy treatment has become an attractive treatment modality in accordance with the development of treatment devices, such as stent retrievers. ${ }^{4}$ We encountered a patient with acute BAO who had the aortic anomaly of an aberrant right subclavian artery (ARSA) in which the right subclavian artery originated from the most distal end of the aortic arch. This particular anomaly has a high incidence among intrathoracic arterial anomalies. ${ }^{5)}$ We report here a rare case of successful endovascular treatment for acute BAO approached via the ARSA.

\section{Case Presentation}

A 66-year-old woman presented to the emergency room with nausea and vomiting, and her consciousness was gradually deteriorating; she presented to the emergency room 1 hour and 15 minutes after symptom onset. Her Glasgow Coma Scale (GCS) score was 8 and her National Institutes of Health Stroke Scale (NIHSS) score was 38. She had a history of atrial fibrillation, but had not been treated with anticoagulants. Diffusion-weighted MRI demonstrated hyperintensity in the right cerebellar hemisphere and the 


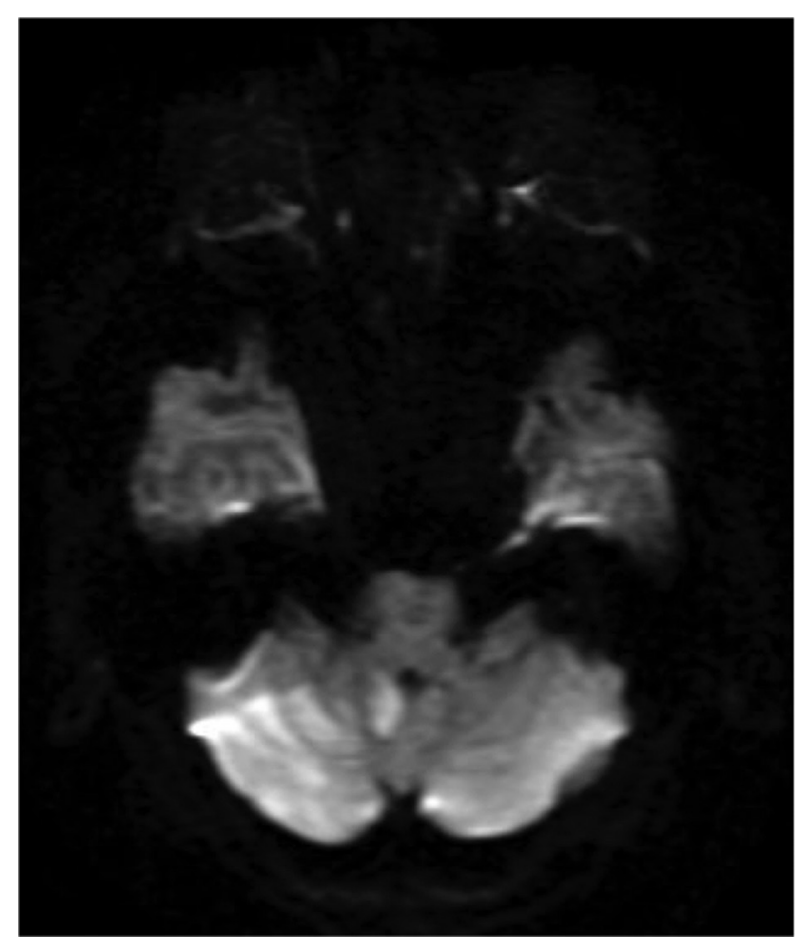

Fig. 1 Pre-treatment diffusion-weighted MRI showing a right cerebellar hemisphere infarction in the right posterior inferior cerebellar artery region.

vermis of the cerebellum in the right posterior inferior cerebellar artery region (Fig. 1). The posterior circulation Acute Stroke Prognosis Early CT score (pc-ASPECTS) ${ }^{6}$ was 9. Magnetic resonance angiography showed a lack of flow in the proximal portion of the basilar artery. Chest radiographs showed no abnormalities. She met the inclusion criteria for intravenous thrombolysis, and rt-PA was administered at 2 hours and 20 minutes after the development of her symptoms.

Despite treatment with intravenous rt-PA, her neurological condition did not improve. Hence, we performed endovascular thrombectomy for acute $\mathrm{BAO}$. The procedure was performed under local anesthesia. A 6-F sheath was placed in the right common femoral artery 3 hours and 44 minutes after symptom onset. We tried to advance a 6-French Roadmaster (Goodman, Aichi, Japan) as a guiding catheter to the right vertebral artery. The right vertebral artery usually originates from the right subclavian artery that arises from the brachiocephalic artery. We advanced the catheter to the artery that we erroneously considered the brachiocephalic artery. However, only the right common carotid artery could be identified. We suspected an aortic anomaly, and therefore, the guiding catheter was inserted in the ascending aorta and a contrast agent was injected (Fig. 2). We found that the right subclavian artery originated from the

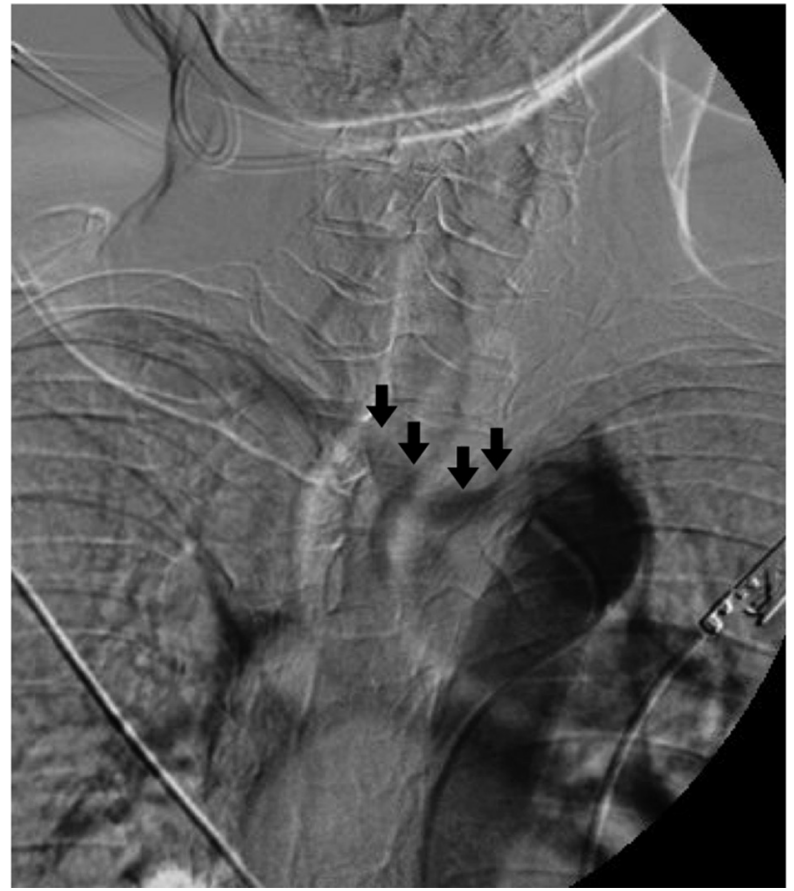

Fig. 2 Anteroposterior digital subtraction view of the contrast agent injected from the ascending aorta. Arrows show the aberrant right subclavian artery.

most distal end of the aortic arch through which the guiding catheter was inserted further into the right vertebral artery.

A Trevo Pro 18 microcatheter (Stryker Neurovascular, Kalamazoo, MI, USA) was advanced distal to the thrombus into the basilar artery. A Trevo Pro clot retriever $4 \times$ $20 \mathrm{~mm}$ (Stryker) was deployed through the microcatheter into the basilar artery and slowly withdrawn with simultaneous guiding catheter aspiration, resulting in complete basilar artery recanalization (Thrombosis in Cerebral Infarction grade 3) at 4 hours and 39 minutes after symptom onset (Fig. 3).

After the procedure, the patient's consciousness improved significantly. Her NIHSS score was 7 and her GCS score increased to 14, 1 hour after the operation. Her NIHSS score was 6 the day after the operation. Mild left hemiparesis and cerebellar ataxia persisted. MRI performed at 24 hours showed infarction in the right cerebellar hemisphere and new infarction in the right pons and the left cerebellar hemisphere (Fig. 4). In addition, an intracerebellar hemorrhage classified as parenchymal hematoma grade 1 (PH 1) according to Safe Implementation of Thrombolysis in Stroke-Monitoring Study (SITS-MOST) classification $^{7)}$ was detected in the left cerebellar hemisphere. Gradually, her left hemiparesis improved. She was 

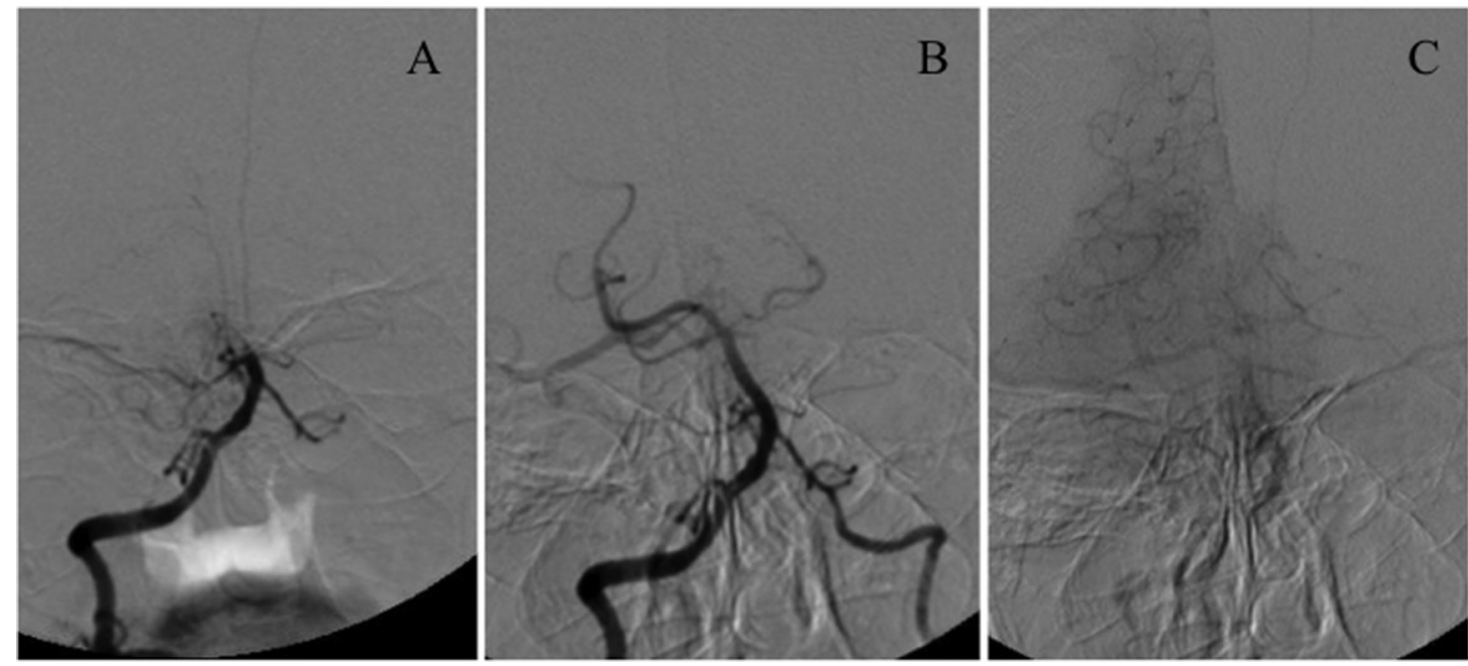

Fig. 3 The pre-treatment digital subtraction view of the right vertebral artery $(\mathbf{A})$ demonstrates complete occlusion of the basilar artery. Early (B) and late (C) arterial phases of the post-treatment view demonstrate complete recanalization of the occlusion.

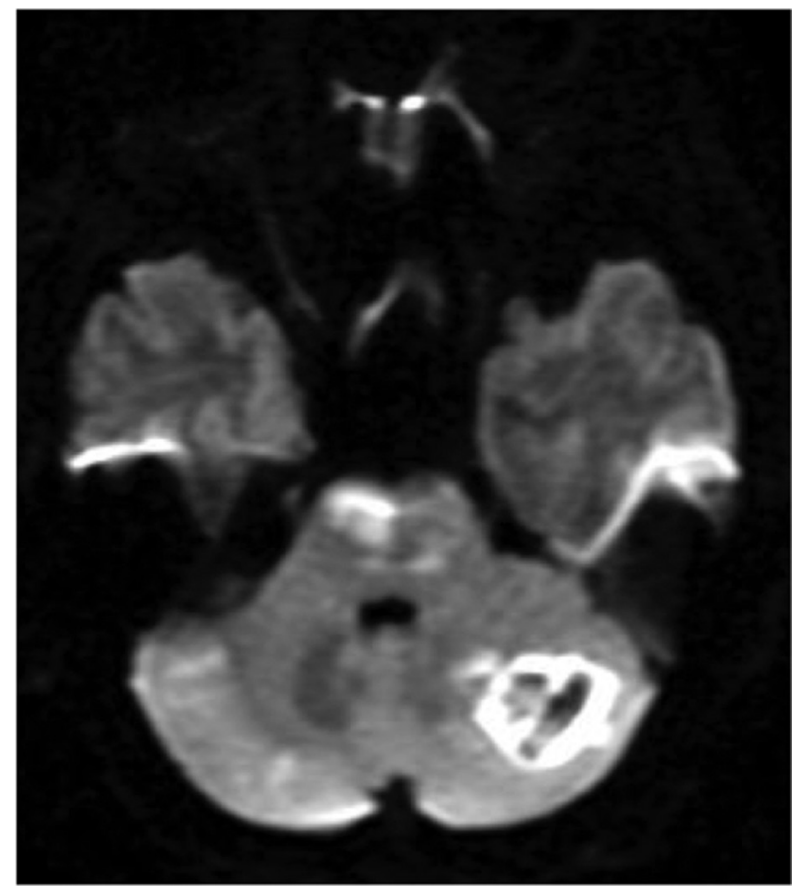

Fig. 4 Post-treatment diffusion-weighted MRI showing infarction in the right pons and left cerebellar hemisphere.

discharged on day 17 with an NIHSS score of 1 . She received anticoagulants (rivaroxaban) for preventing recurrence of the cerebral infarction. Ninety days after treatment, her modified Rankin Scale score was 1. CTA of the chest revealed intrathoracic arterial anomalies, namely the right subclavian artery originated from the most distal end of the aortic arch, that is, a condition known as ARSA (Fig. 5).

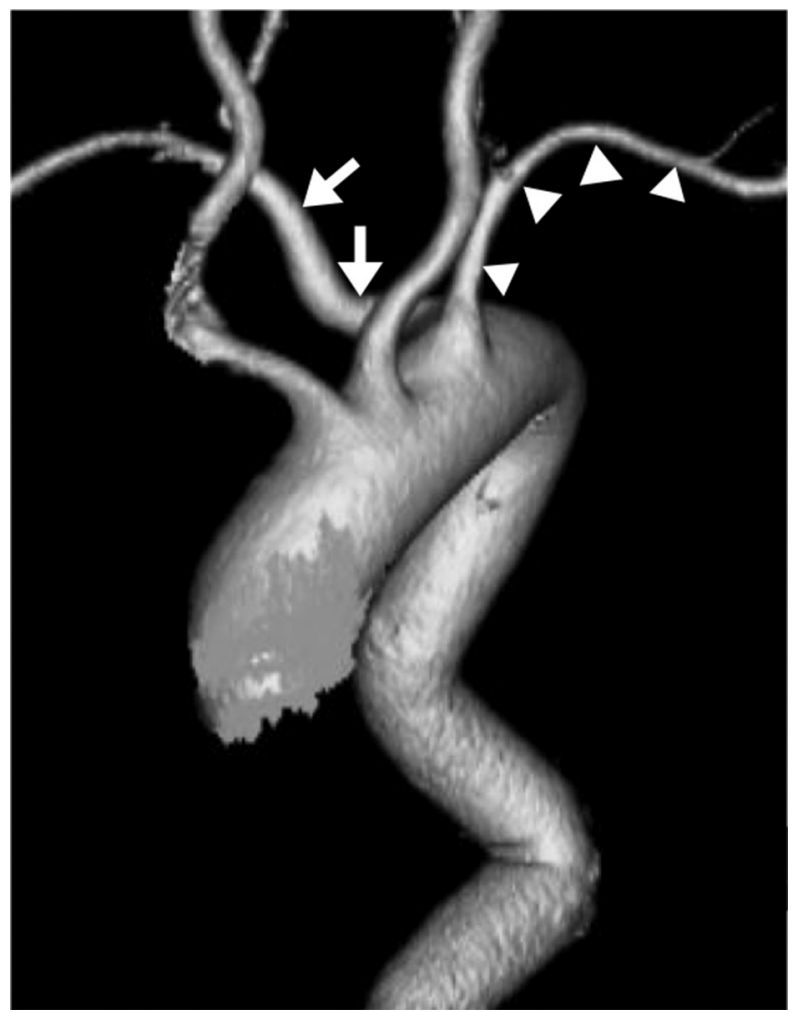

Fig. 5 CT angiography of the thoracic area showing the right subclavian artery originating from the most distal end of the aortic arch. The white arrows and white arrowheads show the aberrant right subclavian artery and left subclavian artery, respectively.

\section{Discussion}

Generally, the basilar artery can be approached via either of the two vertebral arteries. In this case, the right vertebral 
artery was selected to approach the basilar artery because the left vertebral artery was hypoplastic. Although the catheter was advanced to the brachiocephalic artery, the right subclavian artery could not be found. Instead, the right subclavian artery originated from the most distal end of the aortic arch. This condition is termed as ARSA - the most common anomaly of the intrathoracic major arteries with an incidence of $0.4 \%-2 \%{ }^{5}$ ) Common symptoms of ARSA are dysphagia, dyspnea, retrosternal pain, and cough. ${ }^{8)}$ An association between Down syndrome (DS) and ARSA has been suggested, as has an association between feeding problems in young children with DS and ARSA.9) Vascular anomalies, such as truncus bicaroticus and aneurysm, also can coexist with ARSA. ${ }^{8,10)}$

In case of elective treatment, CTA is considered useful to detect ARSA. When this anomaly is recognized before treatment, transradial or transbrachial access of the right side can facilitate the approach to the right vertebral artery. In the present case, we started the endovascular thrombectomy without CTA and selected transfemoral access because urgent reperfusion was necessary. That was the reason for challenging ARSA detection. Once ARSA presence had been recognized, it was easy to advance the guiding catheter to the right vertebral artery arising from the ARSA.

Acute BAO is rare, but associated morbidity and mortality rates are high even with aggressive treatment. ${ }^{1,3,11}$ Although various interventional treatments for acute BAO are available, their validity has not been proved. Schonewille et al. ${ }^{11)}$ showed that patients with a severe neurological deficit had a lower risk of poor outcome after interventional treatment than after conservative therapy. Phan et al. ${ }^{12)}$ demonstrated that stent retriever thrombectomy resulted in a high rate of functional independence. In this case, the patient was in deep coma and had a severe neurological deficit. Therefore, interventional therapy consisting of intravenous rt-PA followed by endovascular thrombectomy was performed to improve the long-term functional prognosis. In this case, we were successful in achieving our aim.

A prolonged duration from stroke onset to recanalization is associated with poor functional outcomes. ${ }^{11,12)}$ Hence, it is necessary to shorten the operation times. However, in some patients with vascular anomalies such as ARSA, the access to intracranial arteries can be delayed. Although intrathoracic arterial anomalies are easily detected by CTA, we use MRI to diagnose acute ischemic stroke. Therefore, we might change the diagnosis of the acute stroke based on the CT image. Moreover, in-depth knowledge of anatomy is a pre-requisite for successful treatment.

\section{Conclusion}

We report a rare case of endovascular treatment for acute $\mathrm{BAO}$. BAO is rare, and the morbidity and mortality rates are very high. Although no evidence has been provided, stent retriever thrombectomy may improve the prognosis of acute BAO. In the course of endovascular treatment, we encountered vascular anomalies. Many anomalies, such as ARSA, hinder the approach to the intracranial arteries; hence, in-depth knowledge of vascular anatomy is imperative.

\section{Funding}

This research received no specific grant from any funding agency in the public, commercial, or not-for-profit sectors.

\section{Disclosure Statement}

The authors declare no conflict of interest.

\section{References}

1) Hornig $\mathrm{CR}$, Lammers $\mathrm{C}$, Büttner $\mathrm{T}$, et al: Long-term prognosis of infratentorial transient ischemic attacks and minor strokes. Stroke 1992; 23: 199-204.

2) Baird TA, Muir KW, Bone I: Basilar artery occlusion. Neurocrit Care 2004; 1: 319-329.

3) Mattle HP, Arnold M, Lindsberg PJ, et al: Basilar artery occlusion. Lancet Neurol 2011; 10: 1002-1014.

4) Yeung JT, Matouk CC, Bulsara KR, et al: Endovascular revascularization for basilar artery occlusion. Interv Neurol 2015; 3: 31-40.

5) Freed K, Low VH: The aberrant subclavian artery. AJR Am J Roentgenol 1997; 168: 481-484.

6) Tei H, Uchiyama S, Usui $T$, et al: Posterior circulation ASPECTS on diffusion-weighted MRI can be a powerful marker for predicting functional outcome. J Neurol 2010; 257: 767-773.

7) Wahlgren N, Ahmed N, Dávalos A, et al: Thrombolysis with alteplase for acute ischaemic stroke in the safe implementation of thrombolysis in stroke-monitoring study (SITS-MOST): an observational study. Lancet 2007; 369: 275-282.

8) Polguj M, Chrzanowski Ł, Kasprzak JD, et al: The aberrant right subclavian artery (arteria lusoria): the morphological and clinical aspects of one of the most important 
variations - a systematic study of 141 reports. Scientific World Journal 2014; 2014: 292734.

9) Roofthooft MT, van Meer H, Rietman WG, et al: Down syndrome and aberrant right subclavian artery. Eur J Pediatr 2008; 167: 1033-1036.

10) Verzini F, Isernia G, Simonte G, et al: Results of aberrant right subclavian artery aneurysm repair. J Vasc Surg 2015; 62: $343-350$.
11) Schonewille WJ, Wijman CA, Michel $P$, et al: Treatment and outcomes of acute basilar artery occlusion in the basilar artery international cooperation study (BASICS): a prospective registry study. Lancet Neurol 2009; 8: 724-730.

12) Phan K, Phan S, Huo YR, et al: Outcomes of endovascular treatment of basilar artery occlusion in the stent retriever era: a systematic review and meta-analysis. $J$ Neurointerv Surg 2016; 8: 1107-1115. 